\title{
Correspondence
}

\section{Loss of the GVL effect by loss of the Y-chromosome as putative mechanism of immune escape in ALL}

\author{
Bone Marrow Transplantation (2005) 35, 101-102. \\ doi:10.1038/sj.bmt.1704712 \\ Published online 1 November 2004
}

Acute lymphoblastic leukemia (ALL) with $\mathrm{t}(9 ; 22)$ is associated with a high frequency of relapse after allogeneic hematopoietic stem cell transplantation. ${ }^{1}$ Despite dismal results of donor lymphocyte infusions (DLI) in ALL, Philadelphia-positive ALL has been associated with a clinical relevant graft-versus-leukemia $(\mathrm{GVL})$ reaction after allogeneic peripheral blood stem cell transplantation (PBSCT). ${ }^{2-4}$ Minor histocompatibility antigens on the Ychromosome (HY) have been identified as important targets for the GVL effect as well as the graft-versus-host disease (GVHD). ${ }^{5}$ Here we report a patient with Philadelphia-positive ALL, who relapsed with loss of the Ychromosome in the relapsed clone after sex-mismatched allogeneic PBSCT and ongoing chronic GVHD.

A 45-year-old male patient (HLA-A*24/-; B*56/-) received a PBSCT from his HLA-identical sister for proB-ALL with $\mathrm{t}(9 ; 22)$ in first partial remission after conditioning with treosulfan and cyclophosphamide. Interestingly, the malignant pro B-ALL cells had myeloid morphology and coexpression of CD34 and CD33 besides otherwise typical surface markers for pro-B-ALL (expression of CD34/CD33/CD19/CD24/CD22/HLA-DR). GVHD prophylaxis consisted of cyclosporin and MTX. He developed acute cutaneous GVHD (grade 2) on day 15 post transplantation, which responded to $2 \mathrm{mg} / \mathrm{kg}$ methylprednisolone. Immunosuppression was stopped at day 70 due to progressive mixed chimerism in the myeloid compartment, while the patient was in molecular remission of ALL. Within a few days after withdrawal of immunosuppression, he developed complete donor chimerism accompanied by steroid-resistant acute GVHD grade 3 involving the skin and gut. Treatment with daclizumab and etanercept resulted in complete resolution of acute GVHD. At 10 months after PBSCT, mbcr-abl (minor-breakpoint) transcripts became detectable again and immunosuppression was tapered. Subsequently, cutaneous lichenoid chronic GVHD developed and molecular remission of mbcr-abl transcripts was shown by nested PCR. At 17 months after PBSCT, chronic lichenoid GVHD progressed to sclerodermoid chronic GVHD. At that time, the bone marrow became mbcr-abl positive again without cytogenetic detection of $t(9 ; 22)$. No male karyotype cells were found in the bone marrow by FISH. Due to local pain in the left knee an MRI was performed, revealing an infiltration of the bone marrow of the left distal femur compatible with a local marrow infiltration with malignant cells. After 2 months (19 months after PBSCT), he developed a hematologic relapse of ALL with the same morphology and expression of identical surface markers (including HLA-DR) as at the time of first diagnosis. At the same time, he had ongoing active chronic sclerodermoid GVHD of the skin, which was confirmed by skin biopsy. FISH for the $\mathrm{X}$ and $\mathrm{Y}$ chromosomes revealed a female phenotype with two $X$ chromosomes and $t(9 ; 22)$ in all evaluated cells. Chimerism analysis of blast cells by PCR of short tandem repeats of chromosomes 4, 8, 18, as well as the $\mathrm{X}$ and $\mathrm{Y}$ chromosomes, detected relapse of recipient origin with loss of the $\mathrm{Y}$ chromosome and exclusive presence of $\mathrm{X}$ chromosomal alleles of the recipient. ${ }^{6}$ Treatment with imatinib was started, but failed to induce any durable cytoreduction. In parallel, further morphologic and phenotypic evolution to a lymphoid morphology and the additional expression of CD10 and CD20 was observed. The patient died after several attempts of cytoreduction 23 months after PBSCT.

The present case demonstrates both the effectiveness and limitations of the GVL effect in Philadelphia-positive ALL. Despite transplantation in partial remission, the patient achieved early molecular remission. Intensified immunosuppression due to GVHD resulted in a molecular relapse, which again resolved in the presence of evolving chronic GVHD. The treatment-resistant relapse of ALL occurred despite active chronic GVHD in the presence of the loss of the $\mathrm{Y}$-chromosome in the malignant cell clone. The most likely explanation for clonal evolution of male ALL to a female karyotype is the selection by an ongoing GVL reaction for the $\mathrm{Y}$-chromosome-associated antigen.

Clonal evolution including loss of chromosomes has been described repeatedly in malignant diseases involving $\mathrm{t}(9 ; 22){ }^{7}$ Although we were not able to prove that a HYassociated antigen was responsible for the observed GVL effect, the coincidence of the loss of the Y-chromosome and the hematologic relapse, as well as the documented role of $\mathrm{HY}$-associated antigens as relevant minor histocompatibility antigens after PBSCT, suggests that the loss of the GVL effect was due to the loss of the coding chromosome. ${ }^{5}$ We hypothesize that the clonal evolution occurred at an immunologically privileged location not accessible to the GVL effect. Subsequent hematologic relapse occurred after loss of the HY antigens coding chromosome. An additional mechanism of immune escape may have been the clonal evolution from myeloid morphology and co-expression of myeloid surface marker to lymphoid morphology, including a new lymphoid surface marker. Myeloid malignant diseases have been associated with a superior sensitivity to the GVL effect in comparison to ALL. ${ }^{8}$
D Wolff ${ }^{1}$
A Knopp ${ }^{1}$
V Weirich ${ }^{2}$
B Steiner ${ }^{1}$
C Junghanss ${ }^{1}$
$\mathrm{J}^{\text {Casper }}{ }^{1}$
M Freund ${ }^{1}$

\author{
${ }^{1}$ Division of Haematology and \\ Oncology, University of \\ Rostock, Germany; and \\ ${ }^{2}$ Institute of Forensic \\ Medicine, University of Rostock, \\ Germany
}




\section{References}

1 Sierra J, Radich J, Hansen JA et al. Marrow transplants from unrelated donors for treatment of Philadelphia chromosomepositive acute lymphoblastic leukemia. Blood 1997; 90: 1410-1414.

2 Collins Jr RH, Goldstein S, Giralt S et al. Donor leukocyte infusions in acute lymphocytic leukemia. Bone Marrow Transplant 2000; 26: 511-516.

3 Keil F, Kalhs P, Haas OA et al. Relapse of Philadelphia chromosome positive acute lymphoblastic leukaemia after marrow transplantation: sustained molecular remission after early and dose-escalating infusion of donor leucocytes. $\mathrm{Br} \mathrm{J}$ Haematol 1997; 97: 161-164.

4 Matsue K, Tabayashi T, Yamada K, Takeuchi M. Eradication of residual bcr-abl-positive clones by inducing graft-versus-host disease after allogeneic stem cell transplantation in patients with
Philadelphia chromosome-positive acute lymphoblastic leukemia. Bone Marrow Transplant 2002; 29: 63-66.

5 Randolph SS, Gooley TA, Warren EH et al. Female donors contribute to a selective graft-versus-leukemia effect in male recipients of HLA-matched, related hematopoietic stem cell transplants. Blood 2004; 103: 347-352.

6 Thiede C, Bornhauser M, Ehninger G. Evaluation of STR informativity for chimerism testing - comparative analysis of 27 STR systems in 203 matched related donor recipient pairs. Leukemia 2004; 18: 248-254.

7 Heerema NA, Harbott J, Galimberti S et al. Secondary cytogenetic aberrations in childhood Philadelphia chromosome positive acute lymphoblastic leukemia are nonrandom and may be associated with outcome. Leukemia 2004; 18: 693-702.

8 Luznik L, Fuchs EJ. Donor lymphocyte infusions to treat hematologic malignancies in relapse after allogeneic blood or marrow transplantation. Cancer Control 2002; 9: 123-137. 